\title{
Brain Imaging of Patients with COVID-19: Findings at an Academic Institution during the Height of the Outbreak in New York City
}

(D)E. Lin, (D).E. Lantos, (DS.B. Strauss, (DC.D. Phillips, (D)T.R. Campion, Jr., (DB.B. Navi, (DN.S. Parikh, (D)A.E. Merkler, (DS. Mir,

(D) C. Zhang, (D) H. Kamel, (D) M. Cusick, (D). Goyal, and (D)A. Gupta

\begin{abstract}
BACKGROUND AND PURPOSE: A large spectrum of neurologic disease has been reported in patients with coronavirus disease 2019 (COVID-19) infection. Our aim was to investigate the yield of neuroimaging in patients with COVID-19 undergoing CT or MR imaging of the brain and to describe associated imaging findings.
\end{abstract}

MATERIALS AND METHODS: We performed a retrospective cohort study involving 2054 patients with laboratory-confirmed COVID-19 presenting to 2 hospitals in New York City between March 4 and May 9, 2020, of whom 278 (14\%) underwent either CT or MR imaging of the brain. All images initially received a formal interpretation from a neuroradiologist within the institution and were subsequently reviewed by 2 neuroradiologists in consensus, with disputes resolved by a third neuroradiologist.

RESULTS: The median age of these patients was 64 years (interquartile range, 50-75 years), and 43\% were women. Among imaged patients, $58(21 \%)$ demonstrated acute or subacute neuroimaging findings, the most common including cerebral infarctions (11\%), parenchymal hematomas (3.6\%), and posterior reversible encephalopathy syndrome (1.1\%). Among the 51 patients with MR imaging examinations, 26 (51\%) demonstrated acute or subacute findings; notable findings included 6 cases of cranial nerve abnormalities (including 4 patients with olfactory bulb abnormalities) and 3 patients with a microhemorrhage pattern compatible with critical illness-associated microbleeds.

CONCLUSIONS: Our experience confirms the wide range of neurologic imaging findings in patients with COVID-19 and suggests the need for further studies to optimize management for these patients.

ABBREVIATIONS: ACE2 = angiotensin converting enzyme 2; COVID-19 = coronavirus disease 2019; MFS = Miller Fisher syndrome; PRES = posterior reversible encephalopathy syndrome; SARS-CoV-2 = Severe Acute Respiratory Syndrome coronavirus 2

S ince the introduction of Severe Acute Respiratory Syndrome coronavirus 2 (SARS-CoV-2) to the human population in December 2019, coronavirus disease 2019 (COVID-19) has rapidly become a global pandemic. While pulmonary manifestations remain its hallmark, COVID-19 is gaining recognition as a multisystem disease, ${ }^{1-6}$ and there is increasing recognition of frequent neurologic manifestations, which reportedly may affect up to

Received June 11, 2020; accepted after revision July 17.

From the Department of Radiology (E.L., J.E.L., S.B.S., C.D.P., A.G.), Department of Population Health Sciences (T.R.C., Jr., M.C.), Clinical and Translational Neuroscience Unit (B.B.N., N.S.P., A.EM., S.M., C.Z., H.K., A.G.), Feil Family Brain and Mind Research Institute and Department of Neurology, and Department of Medicine (P.G.), Weill Cornell Medicine, New York, New York.

E. Lin and J.E. Lantos contributed equally to this article as co-first authors.

This study was supported by National Institutes of Health grants K23NS091395 and R01HL144541. It also received support from NewYork-Presbyterian Hospital and Weill Cornell Medical College, including the Clinical and Translational Science Center (UL1 TR000457) and Joint Clinical Trials Office.

No funding organization had a role in the design and conduct of the study; collection, management, analysis, and interpretation of the data; preparation, review, or approval of the manuscript; or the decision to submit the manuscript for publication.
$36.4 \%$ of patients with COVID-19. ${ }^{7,8}$ While there is growing literature describing neurologic symptoms in patients with COVID19 , little has been published about neuroimaging findings outside of case reports and smaller case series. Our objective was to present the yield of neuroimaging and report the neuroimaging findings in a large cohort of patients with COVID-19 at NewYorkPresbyterian/Weill Cornell Medical Center and NewYorkPresbyterian Lower Manhattan Hospital in New York City.

\section{MATERIALS AND METHODS}

A retrospective cohort study was performed at an academic quaternary-care center and an affiliated community hospital, both in New York City. Using automated systems for electronic capture

Please address correspondence to Eaton Lin, MD, Department of Radiology, Weill Cornell Medicine, 525 East 68th St, New York, NY 10065; e-mail: eal9007@med.cornell.edu

- Indicates open access to non-subscribers at www.ajnr.org

http://dx.doi.org/10.3174/ajnr.A6793 


\begin{tabular}{lcc}
\hline \multicolumn{1}{c}{ Characteristic } & $\begin{array}{c}\text { Patients with COVID-19 } \\
\text { Undergoing CT or MR Imaging of } \\
\text { the Brain ( } \boldsymbol{n}=\mathbf{2 7 8 )}\end{array}$ & $\begin{array}{c}\text { Patients with COVID-19 } \\
\text { without Brain Imaging } \\
(\boldsymbol{n}=1776)\end{array}$ \\
\hline Age (yr) & $71.8(15.4)$ & $60.6(18.1)$ \\
Male & $165(59 \%)$ & $1009(57 \%)$ \\
Race & & \\
Asian & $42(15 \%)$ & $222(13 \%)$ \\
Black & $37(13 \%)$ & $231(13 \%)$ \\
White & $84(30 \%)$ & $524(30 \%)$ \\
Other & $58(21 \%)$ & $400(23 \%)$ \\
Unknown & $57(21 \%)$ & $399(22 \%)$ \\
Medical comorbidities & $79(28 \%)$ & \\
Atrial fibrillation & $214(77 \%)$ & $244(14 \%)$ \\
Hypertension & $115(41 \%)$ & $1030(58 \%)$ \\
Hyperlipidemia & $144(52 \%)$ & $515(29 \%)$ \\
Diabetes & $93(33 \%)$ & $709(40 \%)$ \\
Coronary artery disease & $58(21 \%)$ & $421(24 \%)$ \\
Chronic kidney disease & $40(14 \%)$ & $267(15 \%)$ \\
COPD & $53(19 \%)$ & $150(8 \%)$ \\
Mechanical ventilation & $92(33 \%)$ & $292(16 \%)$ \\
ICU admission & $61(22 \%)$ & $399(22 \%)$ \\
Death & $213(12 \%)$ \\
\hline
\end{tabular}

Note:-COPD indicates chronic obstructive pulmonary disease; ICU, intensive care unit.

${ }^{a}$ Data are reported as number (\%) except where otherwise noted.

${ }^{\mathrm{b}}$ Age is presented as mean age, with SD in parentheses.

of laboratory data, we identified all patients with an active COVID-19 infection with an emergency department visit or hospitalization between March 4, 2020, and May 9, 2020. All COVID-19 diagnoses were confirmed via detection of SARSCoV-2 RNA in nasopharyngeal swab specimens by real-time reverse transcription polymerase chain reaction. The study population was then narrowed to adults (18 years of age or older) who underwent cranial neuroimaging (either CT or MR imaging of the brain) during this period. The racial demographics of our cohort were included given the disproportionate burden of COVID-19 among racial minorities and to assess the generalizability of our findings. Weill Cornell Medicine's COVID-19 Research Data Repository automated data capture feature was also used to collect data on performed imaging studies, demographics, medical comorbidities, presenting symptoms, and the use of mechanical ventilation. ${ }^{9}$ Our institutional review board approved this study and waived the requirement for informed consent.

CT imaging was performed on 1 of 3 scanners: HD Discovery 750, Optima 660, or Revolution EVO (GE Healthcare) with 0.625-mm section thickness, 22 -cm FOV, $120 \mathrm{kV}$ (peak), and 250-300 mA. Coverage was from the foramen magnum to the vertex. Images were reconstructed in the axial plane at 2.5- and 5$\mathrm{mm}$ intervals and in the coronal and sagittal planes at 2-mm intervals. MR imaging was performed on 1 of 3 machines: SIGNA Architect (3T), Discovery 750 (3T), or SIGNA Artist (1.5T) (GE Healthcare). Postcontrast MR imaging was performed using intravenous gadobutrol, $0.1 \mathrm{mmol} / \mathrm{kg}$ (Gadavist; Bayer Schering Pharma). Although there was some variability in MR imaging protocols, all MRIs included diffusion-weighted imaging, SWI, T2 FLAIR, and T1-weighted sequences.

Neuroimaging was reviewed by 2 Certificate of Added Qualification-certified neuroradiologists, each with 10 years of experience. A third Certificate of Added Qualification-certified neuroradiologist was available for resolving discrepancies, but none arose. Neuroimaging findings were documented, including acute or subacute infarctions, posterior reversible encephalopathy syndrome (PRES), and acute or subacute hemorrhage. PRES was determined by the presence of confluent T2-FLAIR hyperintensity or CT hypoattenuation, as described in prior literature. ${ }^{10}$ The burden of microhemorrhage seen on SWI sequences was also recorded. Chronic findings, including chronic infarcts and white matter changes deemed likely of microvascular origin based on age and expected findings, were considered incidental to the event and not recorded. Special attention was directed toward the presence or absence of previously reported COVID-19related neuroimaging findings, including acute hemorrhagic necrotizing encephalopathy, ${ }^{11}$ leptomeningeal enhancement, ${ }^{12}$ cortical T2 hyperintensity, ${ }^{13}$ cranial nerve abnormalities, ${ }^{14}$ and olfactory cleft obstruction. ${ }^{15}$

Stroke mechanisms and etiologies of parenchymal hematomas were determined by 2 study neurologists as per established criteria and classification systems. ${ }^{16-18}$ We used descriptive statistics to characterize the patient cohort and to estimate the yield of neuroimaging. We used STATA (Version 15.1; StataCorp) to perform statistical analyses.

\section{RESULTS}

Our 2 hospitals treated 2054 adult patients with laboratory-confirmed COVID-19 between March 4, 2020, and May 9, 2020, including 263 patients with emergency department visits and 1791 hospitalized patients. Among the total cohort of patients, the age range was $18-101$ years, median age was 64 years (interquartile range, $50-75$ years), and $43 \%$ were women. Among the cohort, 491 (24\%) were admitted to the intensive care unit and 345 (17\%) were mechanically ventilated. Patient demographics and medical comorbidities are further detailed in the Table. Cross-sectional neuroimaging of the brain was performed for 278 (14\%) patients, with 269 (13\%) patients undergoing CT, 51 (2.5\%) patients undergoing MR imaging, and 42 (2.0\%) patients undergoing both CT and MR imaging. For 17 of the 51 patients undergoing an MR imaging examination, imaging was performed both before and after intravenous administration of gadobutrol.

Among the 278 patients with neuroimaging, 58 (21\%) demonstrated acute or subacute neuroimaging findings. There were 31 cerebral infarctions (11\%), 10 parenchymal hematomas (3.6\%), 6 cases of cranial nerve abnormalities (2.2\%), 3 cases of PRES (1.1\%), 3 cases of probable critical illness-associated microhemorrhage (1.1\%), 3 nontraumatic subdural hemorrhages (1.1\%), and 2 


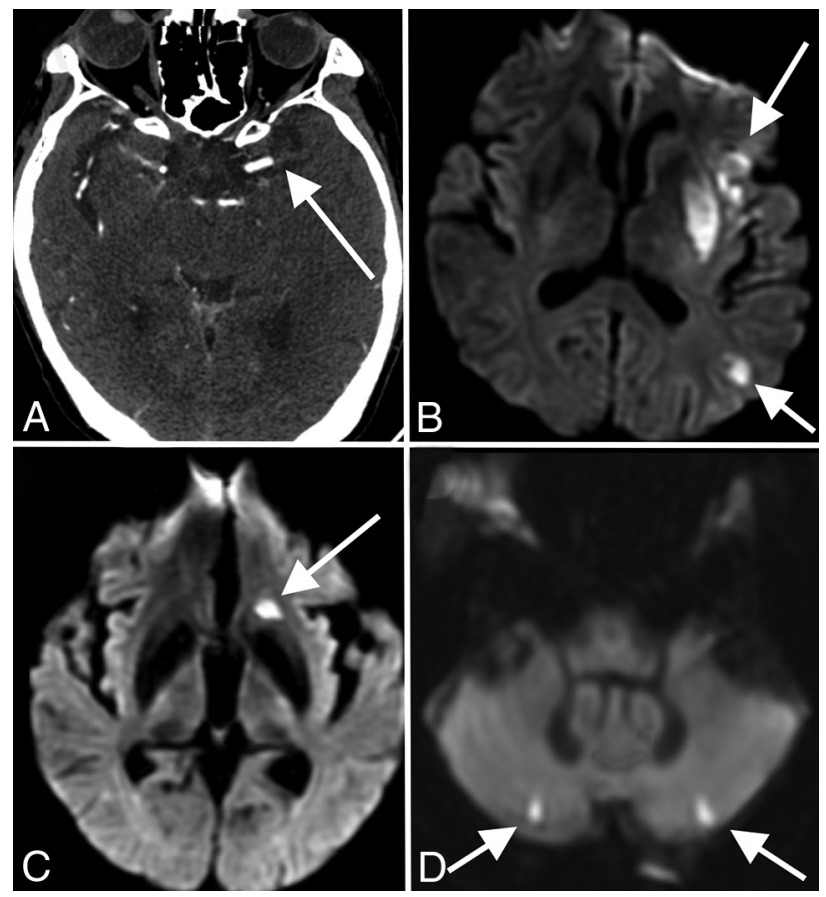

FIG 1. Stroke. A 53-year-old man with COVID-19 with an axial CTA image $(A)$ demonstrating an abrupt cutoff of the proximal $\mathrm{M} 1$ segment of the left MCA (arrow), consistent with thrombosis. The patient later underwent MR imaging, with a DWI sequence (B) demonstrating acute infarctions in the left MCA territory (arrows). An 85year-old woman with COVID-19 and MR imaging with a DWI sequence $(C$ and $D)$ demonstrating acute infarctions in both the anterior (arrow, C) and posterior (arrows, D) circulations, consistent with central embolic etiology. Most patients in our cohort had stroke of either embolic or cryptogenic etiology.

nonaneurysmal subarachnoid hemorrhages $(0.7 \%)$. The yield of neuroimaging was higher among the 51 patients undergoing MR imaging, with 26 (51\%) patients having acute or subacute findings, including 13 cerebral infarctions (26\%), 6 cranial nerve abnormalities (12\%), 3 cases of suspected critical illness-associated microhemorrhage (5.9\%), 2 cases of PRES (3.9\%), and 1 subarachnoid hemorrhage (2.0\%). Individual findings are discussed in more detail below.

\section{Cerebral Infarction}

Acute and subacute infarctions were the most common imaging finding in our cohort (Fig 1). Of 31 patients with cerebral infarctions, the median age was 69 years (interquartile range, 66-78 years) and $13(42 \%)$ were women. Mechanisms of infarction, as determined by our study neurologists, were mostly cryptogenic ( $n=16$, $52 \%)$ or cardioembolic ( $n=13,42 \%)$, with $8(26 \%)$ cases resulting from large-vessel occlusion. Of the cryptogenic infarctions, multiple cerebrovascular territories were involved for 11 (69\%) patients. Of the patients determined to have cardioembolic strokes, 10 had atrial fibrillation, 4 had low ejection fraction heart failure, and 1 was found to have a moderate-to-large patent foramen ovale on an echocardiogram during admission; multiple mechanisms were identified in 2 patients. Aside from the frequency of multiterritory infarctions, no particularly unique imaging characteristics were noted among infarctions in our patients with COVID-19.

\section{Intracranial Hemorrhage}

All except 1 patient with acute parenchymal hematomas in our cohort were men, with a median age of 68 years (interquartile range, 67-74 years). Of the 10 hematomas, $6(60 \%)$ were $>5 \mathrm{~cm}$, all with intraventricular extension, surrounding edema, midline shift, and downward herniation. All 6 of these patients died soon after initial imaging, without follow-up examinations. All 6 patients were on anticoagulation, and 1 had an underlying thrombocytopenia, but only 2 patients demonstrated a hematocrit level on imaging. The remaining 4 cases of parenchymal hematomas either resolved or decreased in size on follow-up imaging. Our study neurologists attributed $6(60 \%)$ cases to anticoagulation, $2(30 \%)$ to indeterminate mechanisms, $1(10 \%)$ to trauma, and $1(10 \%)$ to hypertension. One case of subarachnoid hemorrhage arose from an incidental dural arteriovenous fistula, while the other was posttraumatic. There were also 3 cases of spontaneous subdural hemorrhage.

\section{Microhemorrhages}

Twenty-six (51\%) of the patients having undergone MR imaging had foci of age-indeterminate microhemorrhage. Most $(n=17)$ of these patients had 1-3 foci of microhemorrhage, a number that did not allow further characterization. Seven (14\%) of the patients had a greater burden of microhemorrhage ( $>15$ foci). Of these patients, 1 had a cortical and subcortical distribution sparing the deep gray matter structures in a pattern compatible with cerebral amyloid angiopathy; 1 had predominant involvement of the basal ganglia and cerebellum, with both the microhemorrhage distribution and clinical history compatible with a hypertensive etiology; and 1 had foci attributable to previously treated widespread metastatic disease. Another patient had a nonspecific pattern possibly associated with multiple chronic infarcts but also had a history of hypertension.

The 3 remaining patients with a greater burden of microhemorrhage had innumerable foci, predominantly involving the splenium of the corpus callosum, along with several foci along the remainder of the corpus callosum, internal capsules, and juxtacortical white matter (Fig 2). There were no foci involving the cortex, deep gray matter, brain stem, or cerebellum. The distribution in these 3 patients was atypical for both cerebral amyloid angiopathy and hypertensive microhemorrhage and, instead, was most compatible with critical illness-associated microhemorrhage. $^{19,20}$ All 3 patients had prolonged intensive care unit courses with intubation and mechanical ventilation, but none were treated with extracorporeal membrane oxygenation. Two of the 3 had a history of hypertension, and none of the 3 had a history of seizures or antiepileptic medication use. One patient was receiving therapeutic enoxaparin (Lovenox) for deep vein thrombosis, while the other 2 patients were receiving prophylactic Lovenox at the time of imaging. Initial radiology reporting for 2 of these patients raised the possibility of diffuse axonal injury, but none of these patients had a trauma history. Following extubation, all 3 patients had clinical courses complicated by prolonged delirium and altered mental status. Of the patients with fewer than 15 foci of microhemorrhage, 2 additional patients also demonstrated foci only within the splenium of the corpus callosum.

PRES. There were 3 patients with imaging findings typical of PRES, with acute development of occipital subcortical white 

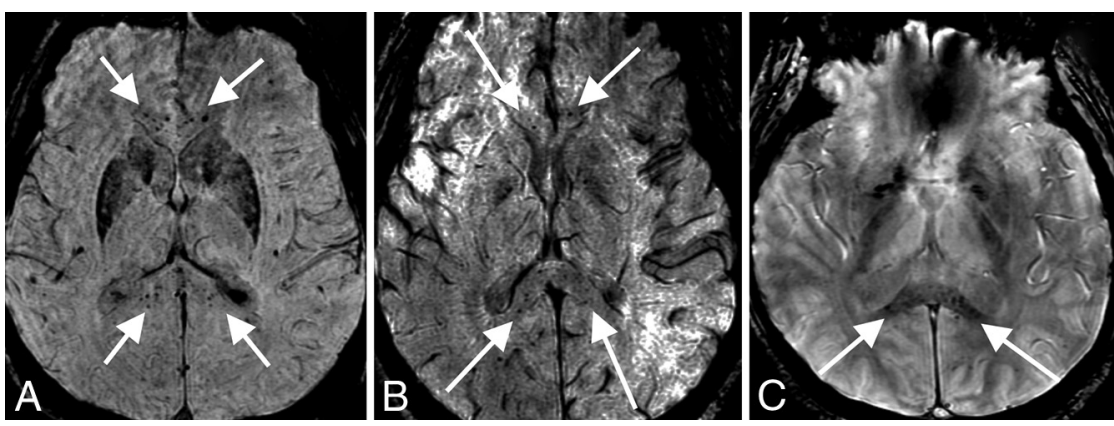

FIG 2. Corpus callosum microhemorrhages. A 65-year-old woman (A), a 44-year-old woman (B), and a 69-year-old man (C) all demonstrate microhemorrhages on SWI, with a similar distribution, preferentially involving the corpus callosum (arrows in $A-C$ ), particularly the splenium. All patients had undergone mechanical ventilation before imaging. The distribution is similar to that previously described in critically ill, ventilated patients as well as in those with high-altitude cerebral edema.

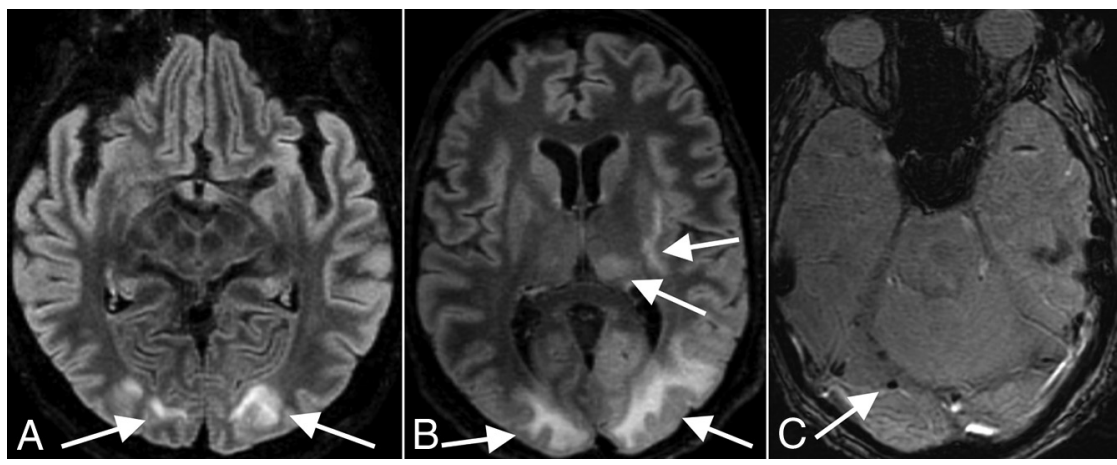

FIG 3. PRES. A 65-year-old woman ( $A$, same patient as in Fig $2 A$ ) and 63-year-old man ( $B$ and $C$ ) demonstrate a typical imaging appearance of PRES on T2-FLAIR images (arrows in $A$ and $B$ ), with bilateral subcortical occipital white matter hyperintense signal, as well as more pronounced involvement of the patient in $B$ with thalamic and internal and external capsule involvement. This patient also has evidence of associated right occipital microhemorrhage (arrow in C). Both patients had the typical risk factors for PRES of acute kidney injury and hypertension.
The first patient presented with diplopia, ataxia, and areflexia typical of Miller Fisher syndrome (MFS), the cranial nerve variant of GuillainBarré syndrome. MR imaging of the brain with dedicated orbital sequences showed an enlarged, T2-hyperintense left oculomotor nerve with marked nerve enhancement after intravenous gadobutrol administration (Fig 4). This patient had negative serum antiganglioside testing, and symptoms improved after intravenous immunoglobulin treatment. Another patient presented with painless diplopia and right abducens palsy, with MR imaging demonstrating enhancement of the bilateral optic nerve sheaths and posterior Tenon capsules. The patient was treated with hydroxychloroquine, and diplopia and abducens palsy resolved after 2 weeks.

\section{Olfactory Bulb Abnormality}

Given the prevalence of anosmia in patients with COVID-19, ${ }^{21-23}$ our department added thin-section coronal T2 imaging through the olfactory bulbs to brain MRIs for all patients with COVID-19 in whom clinical status allowed the additional scan time. A total of 13 examinations (12 patients; mean age, 58 years; 6 women) included both diagnostic olfactory bulb sequences and high-resolution 3D-T2 FLAIR sequences; these studies were included in a sepamatter edema. One patient, a 72-year-old man, was diagnosed solely on the basis of the CT examination. He was intubated for hypoxic respiratory failure and also developed acute kidney injury. The patient was initially hypotensive, requiring pressors, but afterward was intermittently hypertensive. He had follow-up CT showing resolution of occipital white matter edema. The other 2 patients, a 65 year-old woman (Fig 3A) and a 63-year-old man (Fig 3B, -C), both underwent CT and MR imaging and had evidence of associated acute microhemorrhage on susceptibility-weighted MR imaging sequences. Both of these patients also had complications of hypoxic respiratory failure requiring intubation, acute renal failure, and acute hypertension. The 65-year-old woman did not have follow-up imaging, but the 63-year-old man had follow-up CT showing resolution of edema. None of these 3 patients were septic or hyperammonemic, and no cases of PRES were attributable to medications such as various immunosuppressive or chemotherapeutic agents.

\section{Cranial Neuropathies}

Six patients had cranial nerve abnormalities on MR imaging. Four of these patients had olfactory bulb abnormalities and will be discussed separately. The remaining 2 patients are discussed below. rate systematic review (S.B. Strauss, unpublished data, May 2020). Briefly, no patient demonstrated changes in olfactory bulb volume. However, 4 of 12 patients demonstrated abnormally increased olfactory bulb signal on postcontrast T2 FLAIR, which may reflect intrinsic T2 prolongation or, potentially, contrast enhancement (Fig 5). No abnormalities were identified along the olfactory clefts within this cohort to correlate with findings reported in a prior case report of olfactory cleft obstruction in a patient with COVID-19 with anosmia. ${ }^{15}$ One of the 4 patients with olfactory bulb signal abnormality had documented anosmia, but for the other 3 patients, there was no clinical documentation addressing either the presence or absence of anosmia.

\section{Pertinent Negatives}

None of the $51 \mathrm{MR}$ imaging examinations performed in our cohort demonstrated the abnormal cortical T2-FLAIR hyperintensity described in a recent case series. ${ }^{13}$ There were no cases of hemorrhagic encephalitis in our cohort. There were also no cases with abnormal leptomeningeal enhancement among the 17 patients undergoing postcontrast MR imaging. 


\section{DISCUSSION}

COVID-19 is the disease caused by the novel coronavirus SARS-CoV-2, which emerged in December 2019. As the disease has spread, neurologic manifestations have been increasingly described as a contributor to morbidity and mortality. One review of 214 patients found neurologic symptoms, including headache, ataxia, cognitive impairment, anosmia, and stroke, in $36.4 \%{ }^{7}$ Kandemirli et $\mathrm{al}^{13}$ studied 235 patients who were admitted to the intensive care unit for complications of COVID-19 and found that $21 \%$ had neurologic symptoms. Of the symptomatic patients in that study, $54 \%$ underwent MR imaging of the
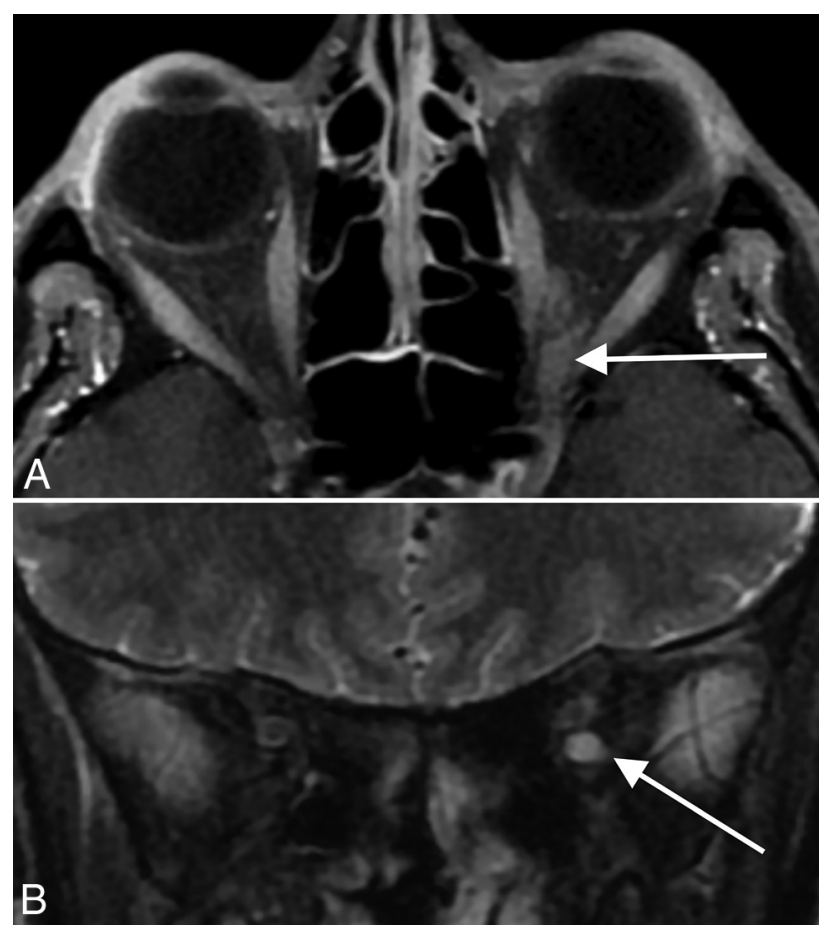

FIG 4. Miller Fisher syndrome. A 36-year-old male patient with a history of COVID-19 and diplopia, ataxia, and areflexia. Axial T1 postcontrast $(A)$ and coronal T2 fat-suppressed $(B)$ MR images through the orbits demonstrate striking enlargement, enhancement, and T2 hyperintense signal of cranial nerve III (arrows in $A$ and $B$ ). The patient was clinically diagnosed with Miller Fisher syndrome and improved with intravenous immunoglobulin treatment.

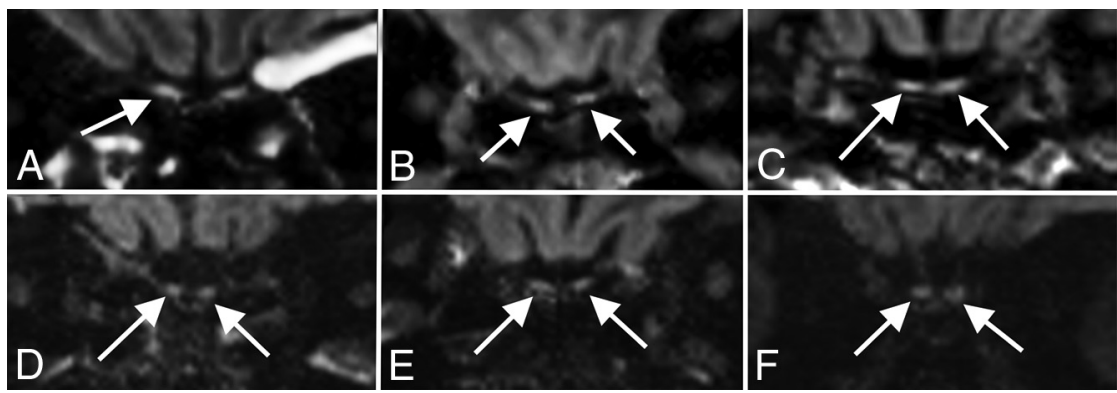

FIG 5. Olfactory neuritis. Six patients with COVID-19 with coronal T2-FLAIR postcontrast images. A 48-year-old woman $(A)$ demonstrates hyperintense signal in the right (arrow) greater than left olfactory bulbs. A 52-year-old woman (B) and a 62-year-old man $(C)$ demonstrate symmetric hyperintense signal in the olfactory bulbs (arrows). For comparison, 3 different patients $(D-F)$ in our cohort with normal olfactory bulbs (arrows) are included. brain, almost half (44\%) of whom had acute findings. Mahammedi et $\mathrm{al}^{24}$ found that $16 \%$ of 725 patients had acute neurologic symptoms and underwent neuroimaging.

At our institution, a review of 2054 patients with COVID-19 presenting during the height of the pandemic in New York City found that 278 patients (14\%) underwent cranial neuroimaging with either CT, MR imaging, or both. Of our imaged patients, $21 \%$ had acute or subacute findings, with a higher yield on MR imaging (51\%). Ischemic stroke (11\%) was the most common acute finding, and most were multifocal and determined to be either cryptogenic or cardioembolic in origin. Potential pathogenesis of stroke in COVID-19 may be from the development of a coagulopathy or endothelial dysfunction. ${ }^{25}$ Coagulopathy may be attributable to the thrombophilic effects of systemic inflammation; the presence of lupus anticoagulant and antiphospholipid antibodies has also been reported. ${ }^{5,25,26}$ Despite reported elevated D-dimer levels and a high incidence of deep vein thrombosis in COVID-19, ${ }^{27}$ we did not find evidence of cerebral venous thrombosis in our cohort. Parenchymal hematomas were found in 10 (3.6\%) patients, most $(n=6)$ of whom had hemorrhages attributed to anticoagulation, a finding that highlights the risks of initiating anticoagulant therapy in response to the prothrombotic features of patients with COVID-19.

Microhemorrhages were found in 26 of 51 patients, with 3 patients demonstrating extensive microhemorrhages predominantly involving the corpus callosum, similar to those shown in a recent case report of a patient with COVID-19 and PRES ${ }^{28}$ and also in keeping with another recent observational study describing 4 patients with COVID-19 with microhemorrhage in the corpus callosum. ${ }^{29}$ This pattern of microhemorrhage involving the corpus callosum has also been described in critically ill patients, including those mechanically ventilated for acute respiratory distress syndrome, as well as in patients with high-altitude cerebral edema. ${ }^{19,30}$ High-altitude cerebral edema microhemorrhages are thought to be due to increased cerebral venous pressure, and positive pressure ventilation may impair cerebral venous return secondary to raised intrathoracic pressure, perhaps explaining the common finding in these 2 distinct patient populations. ${ }^{19}$ All 3 of our patients with callosal microhemorrhage received mechanical ventilation before MR imaging. Therefore, callosal microhemorrhage in COVID-19 is potentially attributable to mechanical ventilation rather than a direct effect from the virus. These 3 patients did not receive extracorporeal membrane oxygenation, which has previously been reported with callosal microhemorrhages. ${ }^{31}$ We agree with Radmanesh et $\mathrm{al}^{29}$ that these callosal hemorrhages resemble those seen with diffuse axonal injury, but, as in their study, the clinical history of our patients excludes posttraumatic etiology.

Our review found 2 types of cases in which neural tissue was involved. The first is a patient previously reported $^{14}$ who presented with clinical MFS and had imaging confirming 
oculomotor nerve abnormality. Serum ganglioside testing was negative in this patient, leaving open the possibility that there was direct inoculation of the nerve by SARS-CoV-2, rather than a cross-reactive immune response induced by molecular mimicry, as is more typical of MFS. MFS is the cranial nerve variant of Guillain-Barré syndrome, which has also been described in COVID-19 in prior case reports, also with negative serum ganglioside testing. ${ }^{32}$ Furthermore, our patient presented with MFS and then tested positive for SARS-CoV-2, indicating that MFS may be a parainfectious rather than postinfectious process, similar to that reported with the Zika virus. ${ }^{33,34}$ Parainfectious Guillain-Barré syndrome has also been recently reported in association with COVID-19. ${ }^{35}$ Whether COVID-19 has the propensity to cause cranial neuropathies more or less than other viruses is an area of potential future investigation and may depend on various factors, including, but not limited to, viral load, neurotropism, and systemic inflammation.

We also found that 4 of our patients with COVID-19 had evidence of an olfactory neuritis, with hyperintensity of the olfactory bulb on postcontrast T2 FLAIR images, potentially a biomarker of disease and an imaging correlate of the anosmia commonly experienced by patients. Recently, it has been shown that sustentacular cells of the olfactory epithelium (technically part of the peripheral nervous system) express the angiotensin converting enzyme 2 (ACE2) receptor, ${ }^{36}$ providing a potential entry point to the CNS via synapses with the olfactory bulbs. Extension to the brain may occur via further trans-synaptic spread, as has been shown to occur in other coronaviruses. ${ }^{37}$ For example, transnasal exposure in mice to the related coronavirus SARS$\mathrm{CoV}$ resulted in detection of the virus in regions with direct neuronal connection to the olfactory bulb: the piriform and infralimbic cortices, basal ganglia, and midbrain. ${ }^{38}$ Another coronavirus, Middle East respiratory syndrome coronavirus, has also been shown to invade the brain of mice when administered intranasally. ${ }^{39}$ Trans-synaptic spread may also allow coronaviruses to gain access to the CNS via other parts of the peripheral nervous system. ${ }^{37}$

Hematogenous spread to the CNS is proposed to be due to viral binding to ACE2 receptors in the capillary endothelium, resulting in damage to the endothelial lining. This could lead to increased permeability of the blood-brain barrier and loss of hemostatic regulation, with resultant cerebral edema. This mechanism could explain the PRES cases that we observed, as has been recently proposed in 2 other cases of PRES with COVID-19. ${ }^{28}$ However, each of our 3 patients with PRES also had the typical risk factors of acute kidney injury and elevated blood pressure. Endothelial damage could also allow the SARS-CoV-2 spike protein to bind directly with neural ACE2 receptors. However, $\mathrm{Li}$ et $\mathrm{al}^{37}$ believed that the related virus SARS-CoV is highly unlikely to spread to the brain by the hematogenous or lymphatic route because almost no viral particles have been detected in nonneuronal cells of infected brain areas in multiple studies. By implication, the same could be true of SARSCoV-2.

Poyiadji et $\mathrm{al}^{11}$ were the first to describe acute hemorrhagic necrotizing encephalitis in the setting of COVID-19 infection. We did not have a similar case in our relatively large cohort, indicating that this may be an uncommon neuroimaging finding, but further investigation is required to assess the incidence in association with COVID-19. Our study did not confirm the cortical T2-FLAIR signal abnormality recently reported in 10 of 27 patients admitted to the intensive care unit for COVID-19. ${ }^{13} \mathrm{We}$ also did not observe abnormal leptomeningeal enhancement in any of our patients undergoing postcontrast imaging, despite prior reports of this finding in COVID-19. ${ }^{12}$

Limitations of this study include those inherent in a retrospective, observational design. In addition, our patients are from 2 centers during the height of the pandemic in New York City and may not represent the true spectrum of neuroimaging findings in COVID-19, especially more chronic, long-term findings. Some of our patients with relatively mild neurologic symptoms may not have undergone imaging due to regulatory constraints imposed during the pandemic, and on the contrary, some patients with more pronounced neurologic impairment may have been too unstable to undergo imaging. Furthermore, it is uncertain whether the observed findings are directly related to SARS-CoV2 infection rather than to coincident conditions, particularly because the patients undergoing imaging had more medical comorbidities than the overall cohort. Given the retrospective design and the critically ill conditions of many of these patients, it was also difficult to obtain full clinical correlation for many of the patients.

Finally, our study was not comprehensive and omitted a few potential areas of interest. For example, we did not include pediatric patients in our cohort, but with the emergence of pediatric COVID-19 manifestations such as multisystem inflammatory syndrome, neuroimaging findings in this population may be of interest. Also, with growing speculation that COVID-19 may be a vascular disease with manifestations in various highly perfused organs, ${ }^{40}$ intracranial vasculitis would be a plausible manifestation of infection; however, due to the limited number and quality of MR angiographic studies performed with a vessel wall imaging protocol in our cohort, we were unable to confidently assess the prevalence of intracranial vasculitis.

\section{CONCLUSIONS}

Understanding of the novel coronavirus SARS-CoV-2 is still in its infancy. Neurologic complications are increasingly recognized, and familiarity with imaging findings is important for radiologists as the pandemic spreads. Of our patients with COVID-19 undergoing neuroimaging, $21 \%$ had acute or subacute findings, including $51 \%$ of the patients undergoing MR imaging. We observed ischemic strokes and intracranial hemorrhages that are similar in appearance to those in patients without COVID-19, though seemingly more common in this patient population. We also observed microhemorrhages with a predilection for the corpus callosum, similar to those previously described in high-altitude cerebral edema and severely ill, ventilated patients. A small subset of our patients had abnormal olfactory nerves, a possible imaging correlate to frequently reported anosmia. Our experience indicates a wide range of neuroimaging findings in COVID-19 and suggests the need for further studies to optimize management of patients with COVID-19 with neurologic manifestations. 
Disclosures: Eaton Lin—RELATED: Grant. National Institutes of Health, Comments: grants K23NS091395 and R01HL144541, awarded to one of our authors.* Thomas R. Campion, Jr-RELATED: Grant: National Center for Advancing Translational Sciences, Comments: The Clinical and Translational Science Center (UL1 TR000457) provided funding for the informatics infrastructure that supported this study.* Babak B. NaviUNRELATED: Expert Testimony: medicolegal consulting on stroke; Grants/Grants Pending: National Institutes of Health grants K23NS091395 and R01HL144541; Other: Data Safety and Monitoring Board Member for Patient-Centered Outcomes Research Institute-funded TRAVERSE trial. Neal S. Parikh-RELATED: Grant: Leon Levy Foundation, Florence Gould Foundation, New York State Empire Clinical Research Investigator Program, Comments: The funding was not specific to this project but provides salary support for my research time.* Alexander E. Merkler-UNRELATED: Expert Testimony: medicolegal consulting on neurological disorders; Grants/Grants Pending: American Heart Association, Comments: American Heart Association grant 18CDA34110419. Hooman Kamel—OTHER RELATIONSHIPS: Dr Kamel serves as coPrincipal Investigator for the National Institutes of Health-funded ARCADIA trial (National Institute of Neurological Disorders and Stroke U01NS095869), which receives an in-kind study drug from the Bristol Myers Squibb-Pfizer Alliance for Eliquis and ancillary study support from Roche Diagnostics; serves as Deputy Editor for JAMA Neurology; serves as a steering committee member of the Medtronic Stroke AF trial (uncompensated); serves on an endpoint adjudication committee for a trial of empagliflozin for Boehringer Ingelheim; and has served on an advisory board for Roivant Sciences related to factor XI inhibition. Ajay Gupta-RELATED: Grant: National Institutes of Health*; UNRELATED: Travel/Accommodations/Meeting Expenses Unrelated to Activities Listed: GE Healthcare and Siemens USA. *Money paid to the institution.

\section{REFERENCES}

1. Oudkerk M, Büller HR, Kuijpers D, et al. Diagnosis, prevention, and treatment of thromboembolic complications in COVID-19: report of the National Institute for Public Health of the Netherlands. Radiology 2020 Apr 23. [Epub ahead of print] CrossRef Medline

2. Goyal P, Choi JJ, Pinheiro LC, et al. Clinical characteristics of Covid19 in New York City. N Engl J Med 2020;382:2372-74 CrossRef Medline

3. Zheng YY, Ma YT, Zhang JY, et al. COVID-19 and the cardiovascular system. Nat Rev Cardiol 2020;17:259-60 CrossRef Medline

4. Klok FA, Kruip M, van der Meer NJM, et al. Incidence of thrombotic complications in critically ill ICU patients with COVID-19. Thromb Res 2020 Apr 10. [Epub ahead of print] CrossRef Medline

5. Helms J, Tacquard C, Severac F, et al. CRICS TRIGGERSEP Group (Clinical Research in Intensive Care and Sepsis Trial Group for Global Evaluation and Research in Sepsis). High risk of thrombosis in patients with severe SARS-CoV-2 infection: a multicenter prospective cohort study. Intensive Care Med 2020;46:1089-98 CrossRef Medline

6. Fukuhara S, Rosati CM, El-Dalati S. Acute type A aortic dissection during COVID-19 outbreak. Ann Thorac Surg 2020 Apr 22. [Epub ahead of print] CrossRef Medline

7. Mao L, Jin H, Wang M, et al. Neurologic manifestations of hospitalized patients with coronavirus disease 2019 in Wuhan, China. JAMA Neurol 2020;77:683. Apr 10. [Epub ahead of print] CrossRef Medline

8. Wu Y, Xu X, Chen Z, et al. Nervous system involvement after infection with COVID-19 and other coronaviruses. Brain Behav Immun 2020;87:18-22 CrossRef Medline

9. Sholle ET, Kabariti J, Johnson SB, et al. Secondary use of patients' electronic records (SUPER): an approach for meeting specific data needs of clinical and translational researchers. AMIA Annu Symp Proc 2017;2017:1581-88 Medline

10. Schweitzer AD, Parikh NS, Askin G, et al. Imaging characteristics associated with clinical outcomes in posterior reversible encephalopathy syndrome. Neuroradiology 2017;59:379-86 CrossRef Medline

11. Poyiadji N, Shahin G, Noujaim D, et al. COVID-19-associated acute hemorrhagic necrotizing encephalopathy: CT and MRI features. Radiology 2020;296:E119-20 CrossRef Medline

12. Helms J, Kremer S, Merdji H, et al. Neurologic features in severe SARS-CoV-2 infection. $N$ Engl J Med 2020;382:2268-70 CrossRef Medline
13. Kandemirli SG, Doagn L, Sarikaya ZT, et al. Brain MRI findings in patients in the intensive care unit with COVID-19 infection. Radiology 2020 May 8. [Epub ahead of print] CrossRef Medline

14. Lantos JE, Strauss SB, Lin E. COVID-19 associated Miller Fisher syndrome: MRI findings. AJNR Am J Neuroradiol 2020;41:1184-86 CrossRef Medline

15. Eliezer M, Hautefort C, Hamel A, et al. Sudden and complete olfactory loss function as a possible symptom of COVID-19. JAMA Otolaryngol Head Neck Surg 2020 Apr 8. [Epub ahead of print] CrossRef Medline

16. Adams HP, Bendixen BH, Kappelle LJ, et al. Classification of subtype of acute ischemic stroke; definitions for use in a multicenter clinical trial-TOAST. Trial of Org 10172 in Acute Stroke Treatment. Stroke 1993;24:35-41 CrossRef Medline

17. Hart RG, Diener HC, Connolly SJ. Embolic strokes of undetermined source: support for a new clinical construct-authors' reply. Lancet Neurol 2014;13:967 CrossRef Medline

18. Meretoja A, Strbian D, Putaala J, et al. SMASH-U: a proposal for etiologic classification of intracerebral hemorrhage. Stroke 2012;43:259297 CrossRef Medline

19. Riech S, Kallenberg K, Moerer O, et al. The pattern of brain microhemorrhages after severe lung failure resembles the one seen in highaltitude cerebral edema. Crit Care Med 2015;43:e386-89 CrossRef Medline

20. Fanou EM, Coutinho JM, Shannon P, et al. Critical-illness associated cerebral microbleeds. Stroke 2017;48:1085-87 CrossRef Medline

21. Vaira LA, Salzano G, Deiana G, et al. Anosmia and ageusia: common findings in COVID-19 patients. Laryngoscope 2020;130:1787CrossRef Medline

22. Gane SB, Kelly C, Hopkins C. Isolated sudden onset anosmia in COVID-19 infection: a novel syndrome? Rhinology 2020;58:299301 CrossRef Medline

23. Hopkins C, Surda P, Kumar BN. Presentation of new onset anosmia during the COVID-19 pandemic. Rhinology 2020;58:295-98 CrossRef Medline

24. Mahammedi A, Saba L, Vagal A, et al. Imaging in neurological disease of hospitalized COVID-19 patients: an Italian multicenter retrospective observational study. Radiology 2020 May 21. [Epub ahead of print] CrossRef Medline

25. Becker RC. COVID-19 update: Covid-19-associated coagulopathy. J Thromb Thrombolysis 2020;50:54-67 CrossRef Medline

26. Zhang Y, Xiao M, Zhang S, et al. Coagulopathy and antiphospholipid antibodies in patients with Covid-19. N Engl J Med 2020;382: e38 CrossRef Medline

27. Tang N, Li D, Wang X, et al. Abnormal coagulation parameters are associated with poor prognosis in patients with novel coronavirus pneumonia. J Thromb Haemost 2020;18:844-47 CrossRef Medline

28. Franceschi AM, Ahmed O, Giliberto L, et al. Hemorrhagic posterior reversible encephalopathy syndrome as a manifestation of COVID-19 infection. AJNR Am J Neuroradiol 2020;41:1173-76 CrossRef Medline

29. Radmanesh A, DermanA Liu YW, et al. COVID-19-associated diffuse leukoencephalopathy and microhemorrhages. Radiology 2020 May 21. [Epub ahead of print] CrossRef Medline

30. Kallenberg K, Dehnert C, Dörfler A, et al. Microhemorrhages in nonfatal high-altitude cerebral edema. J Cereb Blood Flow Metab 2008;28:1635-42 CrossRef Medline

31. Shah J, Armstrong MJ. Extracorporeal membrane oxygenation: uncommon cause of corpus callosal microhemorrhage. Neurology 2015;84:630 CrossRef Medline

32. Toscano G, Palmerini F, Ravaglia $S$, et al. Guillain-Barré syndrome associated with SARS-CoV-2. N Engl J Med 2020;382:2574-76 CrossRef Medline

33. Parra B, Lizarazo J, Jiménez-Arango JA, et al. Guillain-Barré syndrome associated with Zika virus infection in Colombia. $N$ Engl J Med 2016;375:1513-23 CrossRef Medline

34. Brasil P, Sequeira PC, Freitas AD, et al. Guillain-Barré syndrome associated with Zika virus infection. Lancet 2016;387:1482 CrossRef Medline 
35. Zhao H, Shen D, Zhou H, et al. Guillain-Barre syndrome associated with SARS-CoV-2 infection: causality or coincidence? Lancet Neurol 2020;19:383-84 CrossRef Medline

36. Bilinska K, Jakubowska P, Von Bartheld CS, et al. Expression of the SARS-CoV-2 entry proteins, ACE2 and TMPRSS2, in cells of the olfactory epithelium: identification of cell types and trends with age. ACS Chem Neurosci 2020;11:1555-62 CrossRef Medline

37. Li Y, Bai W, Hashikawa T. The neuroinvasive potential of SARSCoV2 may play a role in the respiratory failure of COVID-19 patients. J Med Virol 2020;92:552-55 CrossRef Medline
38. Netland J, Meyerholz DK, Moore S, et al. Severe acute respiratory syndrome coronavirus infection causes neuronal death in the absence of encephalitis in mice transgenic for human ACE2.J Virol 2008;82:7264-75 CrossRef Medline

39. Li K, Wohlford-Lenane C, Perlman S, et al. Middle East respiratory syndrome coronavirus causes multiple organ damage and lethal disease in mice transgenic for human dipeptidyl peptidase 4 . J Infect Dis 2016;213:712-22 CrossRef Medline

40. Varga Z, Flammer AJ, Steiger P, et al. Endothelial cell infection and endotheliitis in COVID-19. Lancet 2020;395:1417-18 CrossRef Medline 